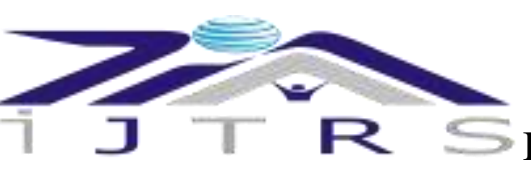

International Journal of Technical Research \& Science

\title{
INTERNET OF THINGS (IOT) AND ITS APPLICATION IN THE REAL WORLD: A STUDY
}

Mohammed Rahmat Ali

E-Mail Id: rahmat_ali2u@yahoo.com

Researcher Scholar, Kulliyah Information Communication Technology- Computer Science, International Islamic University Malaysia (IIUM), Gombak, Malaysia

Abstract-Internets of Things (IoT) a modern buzz word which is acquiring the market with its features and quality which converts the traditional world with the automated world. Internet of things has the next big realistic thing which the world was expecting is turning to be a true reality. Internet of things with emergence in every field has led the users to ease their work with less effort and with maximum outcome. The increase in smarts devices and the decrease in the mobile data charges is the sign how the internet of things is penetrating into the markets and how the number of user increasing day after day. Internet of things is making everything things a digital form.

\section{INTRODUCTION}

Internet of Things is an intelligent device, used to easy the life. Internet of things is the combination of physical device embedded with software and is connected to internet to send, receive, process and to recreate the data. From using the smart phone to launching of the satellite on to the space everything and anything which is connected to the internet is called Internet of Things. Internet of things has emerged in such a way that we knowingly or unknowing are using the technology.

Internet of Technology has helped the user in such that people rely more on devices than their brain. The inclusion of automatic devices and improvement of wireless communication and the usage of smart devices have let the technology to its fullest form of usage. We can monitor anything from anywhere of the globe. Internet of Things has made the life easy and intelligent, with the help of IoT we can assume a smart house, smart car, smart traffic signal, smart industry and smart cities too.

According to a survey analysed be Internet of Things (IoT) Asia, the connected devices by the eng of the year 2020 there will be 6 devices connected to every individual living and is expected to be more in the forth coming time and the acceptance of IoT technology in Asia ranges from 157.8\%, as Singapore to 1.2\%, as Thailand, where as India, stands last second in the list with the adoption of the IoT with readiness of $8.1 \%$ and Malaysia with $34.8 \%$, respectively. Everything is now connected to the internet even the personal devices that we are using shares information with the internet. The best example is GPS, you can be located to anyone anywhere through he/she has not requested to show the availability.

\begin{tabular}{|c|c|}
\hline YEAR & NUMBER OF CONNECTED DEVICES \\
\hline 1990 & 0.3 million \\
\hline 1999 & 90.0 million \\
\hline 2010 & 5.0 billion \\
\hline 2013 & 9.0 billion \\
\hline 2025 & 1.0 trillion \\
\hline
\end{tabular}

\subsection{Internet of Things (IOT) Characteristics}

The key features of Internet of things which makes the technology efficient and effective to use and common to compile are as follows.

\subsection{Artificial Intelligence}

Artificial Intelligence is the branch of science which deals with the artificial brain, which can analyse and take decision, based on the input and can work accordingly. Internet of Things uses the concept of Artificial Intelligence to analyse the decision to be taken, based on the past data. For example considering a smart home technology, which help you when an unknown person enters the hosue without any permission to enter.

\subsection{Connectivity}

Connectivity is the other key factor of Internet of Things, any device which is connected to a network and has a valid IP address can be considered a IoT device. The device will send and receive information from various 


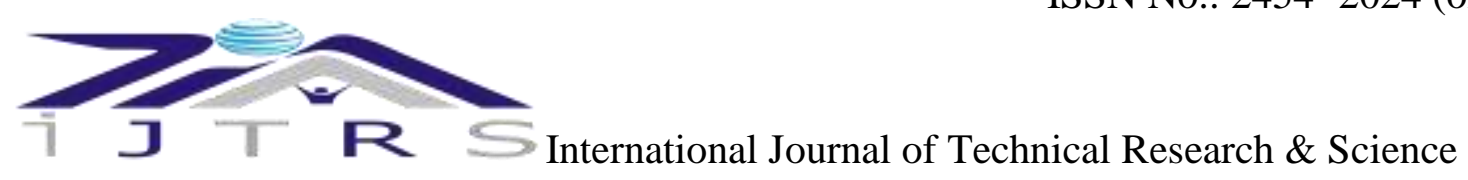

sources and resources. And also it's the responsibility of the device to gather and store information for the further process.

\subsection{Sensors}

Sensors play a prominent role in Internet of Things, Sensors convert one form of signal to another form. Sensors are now used in different applications, such as mobile phone, smart devices, automotive systems, industry controls, health care, oil exploration and climate monitoring etc.,. For example a microphone is a sensor that takes vibration energy (sound waves) and converts it to electrical energy in a useful way for other components in the system to correlate back to the original sound.

\subsection{Active Engagements}

Internet of Things a continuous process of send and receiving of information from various devices and resources to actively participate in the network to communicate from one device to another device. For Example recording the motion and actions of an aeroplane flying high without any major connection to the network.

\subsection{Small Devices}

Internet of things is independent of devices and can work in anywhere. But for efficient work the devices are designed with small size to manage and to carry along. These devices generate information and receive information from other devices.

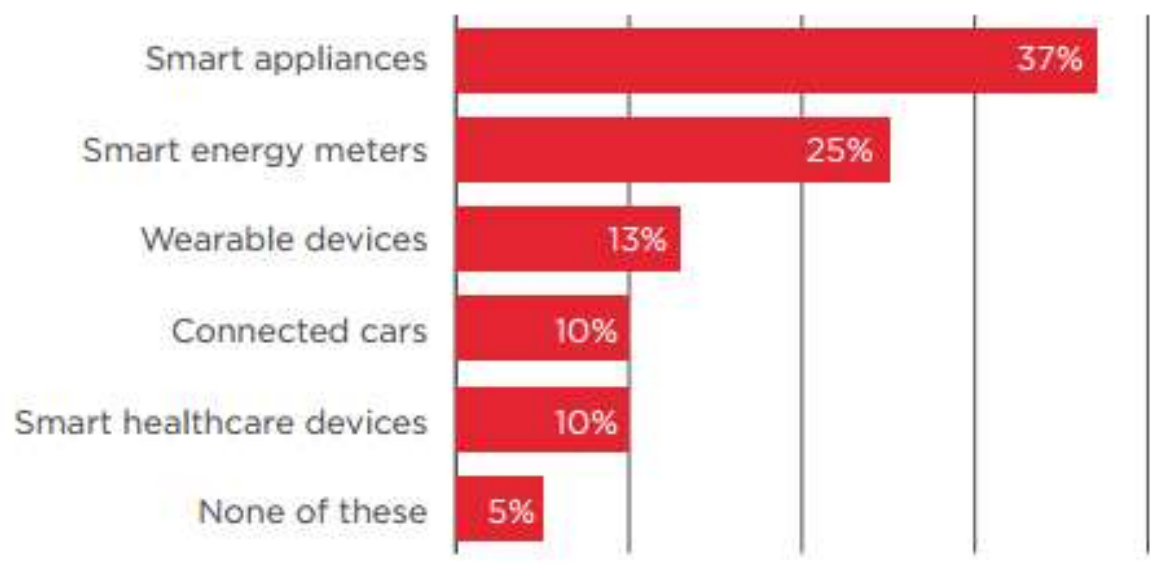

\section{INTERNET OF THINGS HARDWARE}

Internet of Things devices hardware are basically be categorised as wearable and standard devices.

\subsection{Wearable Devices}

The devices which can be carried along with, current wearable smarts devices which can be wore on head are helmet and glasses, which can be wore on neck are jewellery and collars, which can be wore on arms are watches, wristband and rings, which can be wore on feet's are socks and footwear. Apart from wearable IoT devices, there exists some standard devices which includes desktops, tablets and Personal Computers.

\section{INTERNET OF THINGS CLASSIFICATION}

\subsection{Customer to Business or People to Things}

IoT's devices that are either wearable or are through application purpose. The devices such as the health monitoring machines, camera with face deduction, or the cars with sensors are the example of people of things.

\subsection{Machine to Machine or Things to Things}

The things with the interconnections of the object which communicate with one another and make an analysis and give a report are the things to things devices. For example analysis of a medical device to monitor health issue and reports related to it, on a particular patient.

\subsection{Storage in IoT}

The data generated by the devices connected to internet is enormous according a prediction by the end of year 2020 every car will generate two peta byte of data every year, and an aeroplane will generate forty terabyte of data daily. And data that is been generated cannot be transferred instantly on the network, its uses simple memory devices and store the data. Later the data is been uploaded to either the cloud of data ware houses.

\subsection{Common Use of IoT}

IoT is now been used in everywhere, to help, save and ease the work and life style of the people. Internet of Things is being used in engineering, health \& safety, govt sector, medicine. Smart Cities is another powerful 


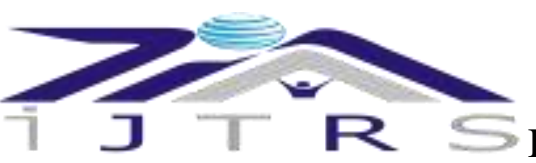

\section{International Journal of Technical Research \& Science}

curiosity that generates among the world's population. Intelligent monitoring, automated transport, smarter energy management systems, water distribution, city security and environmental monitoring are examples of Internet applications for smart cities.

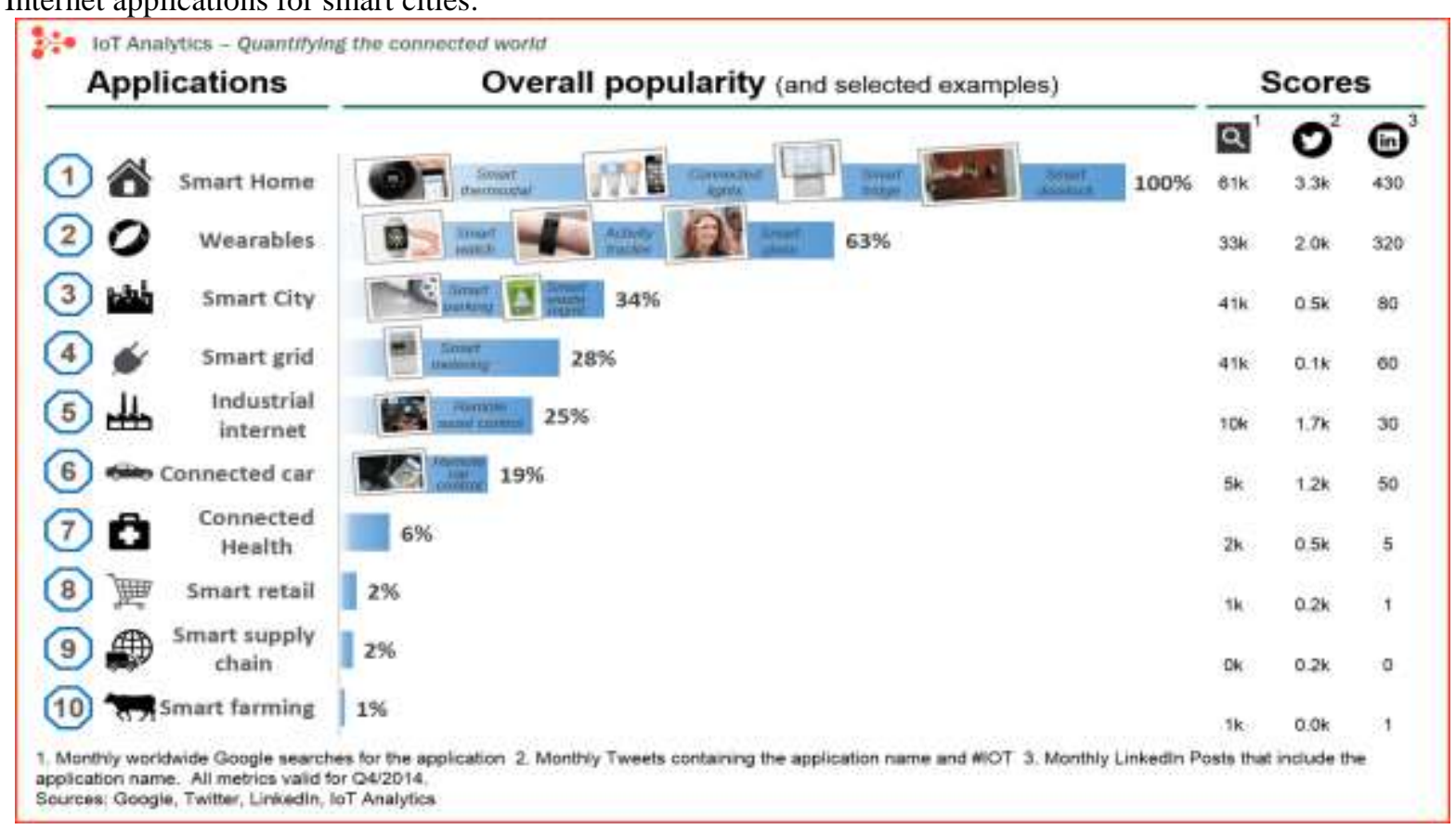

\section{USE OF INTERNET OF THINGS IN MEDIA AND MARKETING}

\subsection{Application Techniques}

The processes in media and advertising involve personal experience that analyzes the system and responds to the needs and interests of each client. This includes general behaviour, our buying habits, your preferences, your culture and other features.

\subsection{Marketing and Content Distribution}

Process technologies operate in a similar and deeper way to the latest data technology, analysis and volume. Existing technologies combine specific data to produce time-related standards and models, but data often lack accuracy and accuracy. Process Technologies Improves this by monitoring more behaviour and analyzing it differently. This leads to more information and details, providing more reliable parameters and reliability. It allows organizations to analyze and respond better to customer needs or priorities. It improves productivity and business strategy and enhances user experience by providing content and related solutions only.

\subsection{Optimize Advertisements}

Advertising is facing excessive and average orientation. Even with today's analysis, recent ads fail. Process Technologies promises different and customized ads from unique strategies. It turns into an advertisement of noise in a practical part of life, where users interact with advertising through operational techniques, not just acceptance. This makes ads more functional and useful to people looking for market solutions or miracles if the solution exists.

\subsection{Use of IoT in Environmental Monitoring}

Environmental monitoring is a widespread application of Internet things. All this allows the monitoring of the ozone level in the meat packing facility to monitor national forest forests. The use of environmental sensors processes techniques for these different applications can take labour-intensive processes and make them easy and efficient.

$>$ Air quality monitoring, carbon dioxide and gas such as smoke and carbon monoxide in confined areas and ozone levels in inland areas.

$>$ Water monitoring of quality, contaminants, thermal contaminants, chemical leaks, presence of lead levels and flood waters.

$>$ Moisture control and ground vibration levels to detect and prevent landslides.

$>$ Monitoring forests and protected areas of forest fires.

$>$ Supervise natural disasters such as earthquake and tsunami.

$>$ Monitoring of hunting for animals and poaching.

$>$ Monitor snowstorms at ski resorts and state forests to monitor weather and sanitation. 


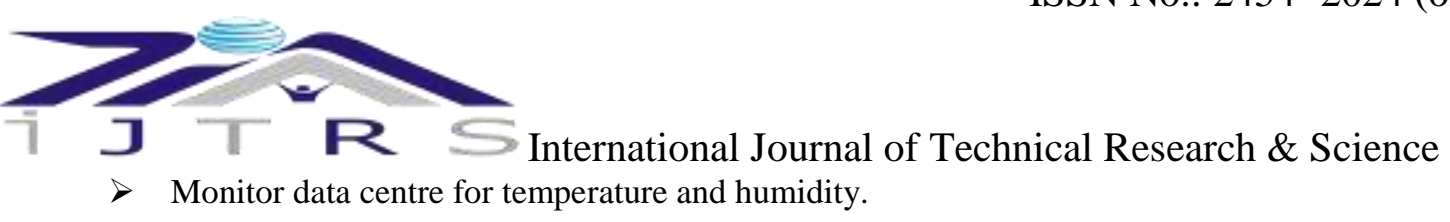

\subsection{Use of IoT in Manufacturing}

The Internet of Things has changed business models and enhances production and automation processes in many industries. But no other sector is more affected by this technological revolution than manufacturing. Manufacturers in all fields of automobile, chemical, durable goods, electronics, etc., have invested in operations techniques and already enjoy benefits. Manufacturers are using Internet solutions that have seen things in 2014 increase in average yield by $28.5 \%$ between 2013 and 2014, according a survey of Tata Consulting.

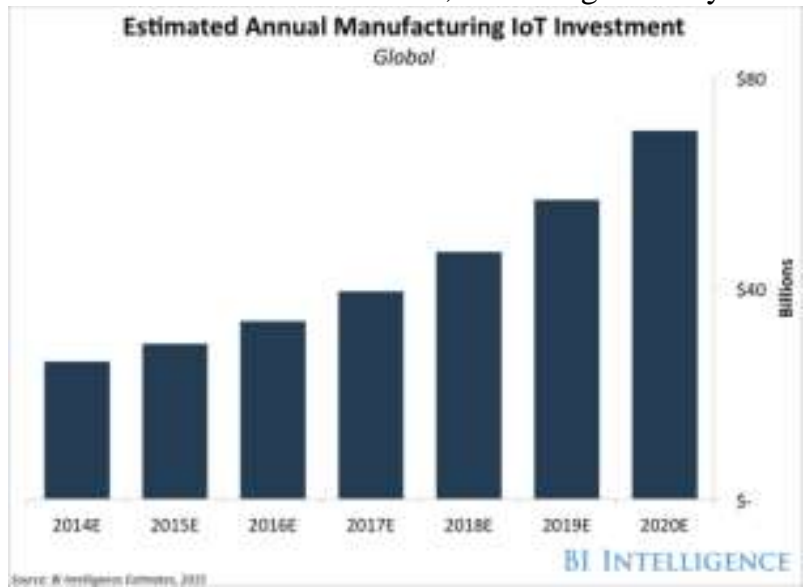

$>$ The Following aims can be achieved with IoT in manufacturing like

$>$ Intelligent Product Enhancements

$>$ Dynamic response to market demand

$>$ Lower cost, optimised resources use and waste reduction

$>$ Improved facilities and safety measure

$>$ Product safety.

IoT is accepted as the intelligent device which can receive and analyse and generate the outcome of the process, industries are accepting the role of IoT to reduce their efforts and to increase the productivity and profitability and can produce good according to the requirement of the customers which saves time and material of the industry.

\subsection{Use of IoT in Energy Application}

Internet of things can manage the energy efficiency, by its low energy consumption and to manage the devices connected to it for the fluctuation of power low or high. Its indicated the devices been repaired or to be replaced.

\subsection{Residential Energy}

IoT devices manage the power consumption and allow the devices to use the amount of power utilised by the day and night. It also replicates the power consumption with reference to the light and power required by brightening or by dimming the light. It also saves the power by turning off the portion of light which is not being used or was not been used for certain amount of time.

\subsection{Commercial Energy}

IoT helps the main firm to save the energy and to increase the productivity of the unit by utilising the amount of power used to manufacture the required items and to save the extra consuming power. Commercially the amount of energy being wasted is more when compared to the amount of energy being used. This is the major concern of power consumption in term of energy and cost. IoT devices helps in not only saving the energy but also using the minimal power required based on the previous consumption.

\subsection{Reliability}

The analysis and procedures provided by the Internet also contribute to system reliability. Behind Use, IoT prevents loading or system bottlenecks. It also detects system threats and Performance and stability, which protects from loses such as downtime, damaged equipment and injuries.

\subsection{Use of IoT in Health Care Application}

Internet of Things conditions in health care (industry, personal health care and health care claims) have increased dramatically through different cases of specific use of Internet material. At the same time, we see how the use of other IoT cases change and the ongoing health reality is accelerating, despite the obstacles that still occur. IoT can help in monitoring the health of an individual and also can let the doctor know about your health. It also helps the doctor to analyse the patient's health and prescribe a proper medicine. It also helps the patient to intimate the condition to the doctor and to hospital to avoid delay in the treatment. 


\section{T证s \\ 4.11 Use of IoT in Building and Housing Application}

IoT is used for a variety of buildings and structures that enable us to automate routine and business routines and needs in a way that dramatically improves the environment and work. This is seen in manufacturing and energy applications, reducing costs, improving security, increasing individual productivity and improving quality of life.

In particular, Internet of things (techniques operations) had a significant impact on Commercial real estate industry, helping the company exceed cost reduction targets. IoT's application is aimed at increasing margins and enabling functions such as more efficient construction processes, better tenant relationships and new revenue opportunities. Consider the increasingly popular smart heat that you adjust intuitively temperature, humidity and light depending on population choices and weather conditions.

Although users of hardware processes operations attracted the most media, is the use of technology in the company is likely to have a significant impact on the industry. In fact, the Commercial Real Estate industry may be in a unique position to implement this technology, using the Buildings Management System which is compatible with Process Technologies to make performance construction and data generated by the sensors to enhance the user more efficiently. Forecasts of smart cities Gartner highlight the potential: "The commercial building of the smart phone become the largest Internet things (operations technologies) until 2017, after the smart home will immediately initiate a billion after binding in 2018" does not allow the CRE to reveal a large segment From the population to technology processes techniques, but also give an opportunity for the owners to talk and direct relationship with the consumer building example, the business centre sensors can help homeowners communicate directly and provide services to the end consumer, which will lead to customer relationships and increased tenant participation.

\section{IOT SECURITY}

As IoT is connected to a network the possibility of attack on the network is also predictable. The attacks can be so serious that, the company might run out of or go bankrupt. Recent times the attack on Ukraine power unit or the attack on cyber security of German steel mill or the attack on the people's security camera to spy on them are the examples.

The security concern can be addressed by the use of smart cards or by using intelligent devices or security chips. Security chip will be embedded with all the information of one that can be used. But the concern is making the chip and testing it and making that chip to only be accessible to the person and thing to be used. Proper measures are been taken into consideration to avoid the security concerns to the IoT, Still a lot of work is still ongoing.

Challenges in Security are

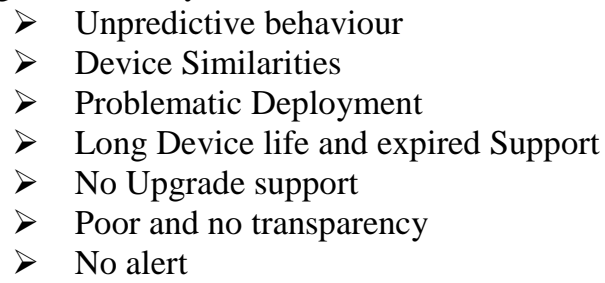

\section{ADVANTAGE OF IOT}

Here are some of the advantages of Internet of Things:

\subsection{Facts}

The more information, the easier to make the right decision. Find out what to do with the grocery store while you are away, without checking for yourself, saving you time, but also easy.

\subsection{Monitoring}

Computers take into account the quality and usefulness of the home. Knowing the product end date before consumption increases the safety and quality of life. In addition, you will not leave without anything when you need it at the last minute.

\subsection{Time}

The elapsed time spent in supervision and the number of trips made otherwise would be great.

\subsection{Money}

The financial aspect is the best advantage. This technology can replace those responsible for monitoring and maintaining supplies.

\section{DISADVANTAGE OF IOT}

The following are some of the weaknesses of Internet things: 


\section{T $\mathrm{R} S$ International Journal of Technical Research \& Science \\ 7.1 Compatibility}

Now there is no standard for labeling and monitoring with sensors. Uniform concepts such as USB or Bluetooth are needed that should not be difficult.

\subsection{Complexity}

Some failures with complex systems. For example, you and your partner may receive a message that the milk is finished, and both are likely to buy the same thing. This leaves you twice the need. Or that there is an error in the program causing the printer to request ink several times when a single cartridge is needed.

\subsection{Confidentiality / Security}

Confidentiality is a big problem with operations techniques. All data must be encrypted so that data about your financial situation or amount of milk you eat is unusual at work or with your friends.

\subsection{Security}

Software may be hijacked and your personal information may be misused. The possibilities are endless. Your change request or details of pirated piracy can cause you risk. Therefore, all safety risks are the responsibility of the user.

\section{CONCLUSION}

IoT is a vast growing field which has been implemented its technology in fast growing industry. Still new sector of societies are implementing the IoT Technology. IoT is the future of the world where once can access the world through intelligent devices. Soon of later the world will be taken by smart and intelligent devices and controlled by internet. The dependency of the user on the internet and the decrease in the data rates are the prominent sigh that the IoT will be the future of the forth-coming world.

\section{REFERENCE}

[1] https://www.analyticsvidhya.com/blog/2016/08/10-youtube-videos-explaining-the-real-world-applicationsof-internet-of-things-iot/

[2] https://iot-analytics.com/10-internet-of-things-applications/

[3] https://www.tutorialspoint.com/internet_of_things/internet_of_things_media_marketing_and_advertising.ht $\mathrm{m}$

[4] http://uk.businessinsider.com/internet-of-things-in-manufacturing-2016-10/?IR=T

[5] http://www.libelium.com/resources/top_50_iot_sensor_applications_ranking/

[6] https://www.i-scoop.eu/internet-of-things-guide/internet-things-healthcare/

[7] https://dupress.deloitte.com/dup-us-en/focus/internet-of-things/iot-commercial-real-estate-intelligentbuilding-systems.html

[8] http://ieeexplore.ieee.org/document/6468307/

[9] http://my.mouser.com/applications/sensor-fusion-iot/

[10] https://en.wikipedia.org/wiki/Internet_of_things

[11] http://internetofthingsagenda.techtarget.com/definition/Internet-of-Things-IoT

[12] Topologies driving IoT networking standards

[13] Internet of Things: Architectures, Protocols, and Applications by Pallavi Sethi and Smruti R. Sarangis

[14] The internet of Everything Protocol and Possible Attacks

[15] Digital Evidences challenges and things by R.C.Hegarty1, D.J.Lamb2 and A.Attwood3

[16] Energy Efficient Network Architecture for loT Application by Sarwesh pi, N.Shekar V. Sheet

[17] Internet of Things-IOT: Definition, Characteristics, Architecture, Enabling Technologies, Application \& Future Challenges

[18] IOT forensics Issue, Strategy and challenges by Jingang Lui

[19] The three software stack requirement for IOT architecture by eclipse.org

[20] Comparative analysis of IoT Architecture by Ravi Teja.

[21] https://www.iso.org/news/2016/09/Ref2112.html

[22] https://dzone.com/articles/iot-systems-sensors-and-actuators

[23] https://www.linkedin.com/pulse/sensors-actuators-iot-mike-vladimer

[24] http://ubiquity.acm.org/article.cfm?id=2822887

[25] https://www.ibm.com/blogs/internet-of-things/cost-effectiveness-sensor-devices/

[26] http://internetofthingsagenda.techtarget.com/definition/IoT-security-Internet-of-Things-security

[27] http://www.mckinsey.com/industries/semiconductors/our-insights/security-in-the-internet-of-things

[28] http://theinternetofthings.report/Resources/Whitepapers/7f4b81fe-25c3-4fa1-a1fba12fa6d42f44_Ayla_Whitepaper_Art-of-IoT-QA.pdf

[29] http://www.gemalto.com/mobile/networks/customer-experience/iot-qos

[30] http://www.ijcee.org/vol8/915-SIM2016-208.pdf

1. http://ieeexplore.ieee.org/document/7800446/

DOI Number: 10.30780/IJTRS.V04.I02.005

www.ijtrs.com

wWw.ijtrs.org 